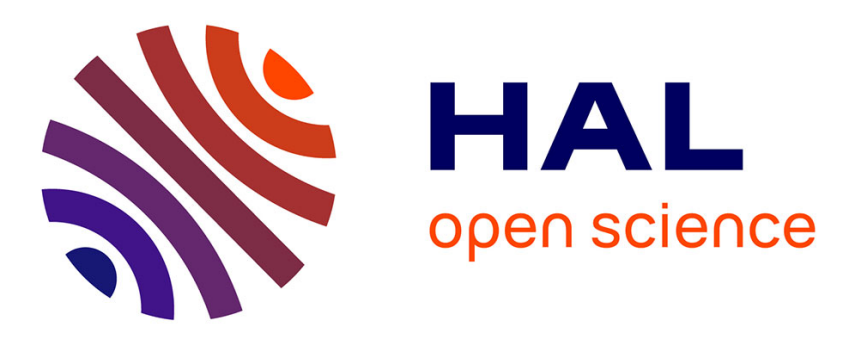

\title{
Robust feedforward boundary control of hyperbolic conservation laws
}

\author{
X. Litrico, Vincent Fromion, G. Scorletti
}

\section{To cite this version:}

X. Litrico, Vincent Fromion, G. Scorletti. Robust feedforward boundary control of hyperbolic conservation laws. 45th IEEE Conference on Decision and Control, Dec 2006, San Diego, United States. pp.5311-5316, 10.1109/CDC.2006.377705 . hal-02588136

\section{HAL Id: hal-02588136 \\ https://hal.inrae.fr/hal-02588136}

Submitted on 15 May 2020

HAL is a multi-disciplinary open access archive for the deposit and dissemination of scientific research documents, whether they are published or not. The documents may come from teaching and research institutions in France or abroad, or from public or private research centers.
L'archive ouverte pluridisciplinaire HAL, est destinée au dépôt et à la diffusion de documents scientifiques de niveau recherche, publiés ou non, émanant des établissements d'enseignement et de recherche français ou étrangers, des laboratoires publics ou privés. 


\title{
Robust feedforward boundary control of hyperbolic conservation laws
}

\author{
Xavier Litrico, Vincent Fromion and Gérard Scorletti
}

\begin{abstract}
The paper proposes a feedforward boundary control to reject measured disturbances for systems modelled by hyperbolic partial differential equations obtained from conservation laws. The controller design is based on frequency domain methods. Perfect rejection of measured perturbations at one boundary is obtained by controlling the other boundary. This result is then extended to design robust open-loop controller when the model of the system is not perfectly known, e.g. in high frequencies. Frequency domain comparisons and time-domain simulations illustrates the good performance of the feedforward boundary controller.
\end{abstract}

\section{INTRODUCTION}

In this paper, we consider the control of plants whose models are hyperbolic partial differential equations obtained from conservation laws, with an independent time variable $t \in[0,+\infty)$ and an independent space variable on a finite interval $x \in[0, L]$. We focus on the design of a feedforward control law in order to reject measured disturbances using boundary control.

The motivation of this work is related to the problem of controlling an open-channel around a given regime, represented by linearized Saint-Venant equations. These hyperbolic partial differential equations describe the dynamics of open-channel hydraulic systems assuming one dimensional flow. This open-loop control problem was first considered in [1], where the authors used a Riemann invariants approach to derive an explicit expression for the open-loop control. The proposed method is however restricted to specific hyperbolic plants corresponding to horizontal frictionless channels.

There are numerous publications related to the design of open-loop controllers for open-channel systems. Most of them are based on simple approximate formulations, see e.g. [9], [2] and references therein.

We use in this paper a frequency domain approach, which enables to extend the results of [1] to the case of canal pools with nonzero slope and friction, and arbitrary uniform geometry (not necessarily rectangular). We design the feedforward compensator that perfectly reject the perturbation. Since the obtained compensator is actually an irrational transfer function, we propose and compare various rational approximations. We also investigate how to design a rational precompensator by explicitly considering the plant uncertainties, that is, a robust feedforward boundary controller.

$\mathrm{X}$. Litrico is with UMR G-EAU, Cemagref, Montpellier, France. xavier.litrico@cemagref.fr

V. Fromion is with INRA-MIG, Domaine de Vilvert, Jouy-en-Josas, France. fromionajouy.inra.fr.

G. Scorletti is with GREYC Equipe Auto, Université de Caen, France. gerard.scorletti@greyc.ensicaen.fr.
The proposed feedforward control is tested in simulation for an open-channel described by linearized Saint-Venant equations where the upstream discharge is the disturbance and the control variable is the downstream discharge.

\section{CONTROL PROBLEM STATEMENT}

We consider a plant modelled by an hyperbolic partial differential equations obtained from conservation laws:

$$
\frac{\partial \xi}{\partial t}+\left(\begin{array}{cc}
0 & 1 \\
\alpha \beta & \alpha-\beta
\end{array}\right) \frac{\partial \xi}{\partial x}+\left(\begin{array}{cc}
0 & 0 \\
-\gamma & \delta
\end{array}\right) \xi=0
$$

where $t$ and $x$ are the two independent variables: a time variable $t \in[0,+\infty)$ and a space variable $x \in[0, L]$ on a finite interval, $\xi(t, x)=(h(t, x), q(t, x))^{T}:[0,+\infty) \times$ $[0, L] \rightarrow \Omega \in \mathbb{R}^{2}$ is the state of the system; $\alpha>\beta>0$, $\gamma \geq 0$ and $\delta \geq 0$ are positive real scalars.

The two equations of system (1) can be interpreted as a mass conservation law with $h$ the conserved quantity and $q$ the flux. The second equation can then be interpreted as a momentum conservation law.

We consider the solutions of the Cauchy problem for the system (1) over $[0,+\infty) \times[0, L]$ under an initial condition $\xi(0, x), x \in[0, L]$ and two boundary conditions of the form $q(t, 0)=q_{0}(t)$ and $q(t, L)=q_{L}(t), t \in[0,+\infty)$.

We assume that the input boundary condition at $x=0$ $q(t, 0)$ is a measured disturbance on the system and that the input boundary condition at $x=L q(t, L)$ is the control variable. The control objective is therefore to design a feedforward boundary controller such that the boundary output variable $h(t, L)$ remains close to 0 . We first discuss the perfect rejection $(h(t, L)=0)$ with the model assumed perfectly known in section III (the feedforward control problem). As the proposed controller is actually irrational, its approximation by a rational transfer function is discussed. In section IV, we no longer assume that the model is perfectly known (uncertain plant). In this case, we investigate how to design a rational compensator (the robust feedforward control problem).

\section{FEEDFORWARD CONTROL DESIGN}

We first compute the input-output transfer matrix of the system, and then derive the exact feedforward controller. The obtained controller, which is infinite dimensional is then decomposed in simple elements.

\section{A. Input-output transfer matrix}

The open-loop transfer matrix of the plant can be obtained by applying Laplace transform to the linear partial differential equations (1), and solving the resulting system of Ordi- 
nary Differential Equations in the variable $x$, parameterized by the Laplace variable $s$ [6].

The downstream water level is then related to upstream and downstream discharges as follows:

$$
h(s, L)=G(s) q(s, 0)+\tilde{G}(s) q(s, L)
$$

with

$$
\begin{aligned}
G(s) & =\frac{\left(\lambda_{2}(s)-\lambda_{1}(s)\right) e^{\left(\lambda_{1}(s)+\lambda_{2}(s)\right) L}}{s\left(e^{\lambda_{2}(s) L}-e^{\lambda_{1}(s) L}\right)} \\
\tilde{G}(s) & =\frac{\lambda_{1}(s) e^{\lambda_{1}(s) L}-\lambda_{2}(s) e^{\lambda_{2}(s) L}}{s\left(e^{\lambda_{2}(s) L}-e^{\lambda_{1}(s) L}\right)}
\end{aligned}
$$

and where the eigenvalues $\lambda_{1}(s)$ and $\lambda_{2}(s)$ are given by, for $i=1,2$ :

$$
\lambda_{i}(s)=\frac{(\alpha-\beta) s+\gamma+(-1)^{i} \sqrt{d(s)}}{2 \alpha \beta}
$$

and $d(s)=(\alpha+\beta)^{2} s^{2}+2[(\alpha-\beta) \gamma+2 \alpha \beta \delta] s+\gamma^{2}$.

This irrational transfer function model (2) gives the frequency domain input-output behavior of the linear hyperbolic system (1) subject to the specified boundary inputs.

\section{B. Feedforward controller design}

1) A naive approach: Using Eq. (2), it is easy to see that an perfect rejection of disturbances provoked by $q(s, 0)$ can be achieved by specifying $q(s, L)$ as follows:

$$
q(s, L)=K_{F}(s) q(s, 0)
$$

with

$$
K_{F}(s)=-\frac{G(s)}{\tilde{G}(s)}=\frac{\left(\lambda_{2}(s)-\lambda_{1}(s)\right) e^{\left(\lambda_{1}(s)+\lambda_{2}(s)\right) L}}{\lambda_{2}(s) e^{\lambda_{2}(s) L}-\lambda_{1}(s) e^{\lambda_{1}(s) L}} .
$$

$K_{F}(s)$ is an irrational controller which is stable. For its implementation, a rational approximation has to be computed.

2) Some practical remarks: In practice, since the system is marginally stable (it contains an integrator), it is not advised to design the open-loop controller represented by eq. (7) and implement it with eq. (6). Indeed, since the system is open-loop marginally stable, any perturbation will destabilize it. Therefore, it is necessary to first stabilize the system with a feedback controller and then to design the open-loop controller for the closed-loop system.

To this purpose, let us assume that the system is stabilized with the following proportional boundary control:

$$
q(s, L)=k_{h} h(s, L)+k_{w} w(s)
$$

where $w$ is an additional boundary input, $k_{h}>0$ and $k_{w} \in$ $\mathbb{R}^{*}$ are real constants ${ }^{1}$.

The resulting closed-loop system becomes:

$$
h(s, L)=G_{1}(s) q(s, 0)+G_{2}(s) w(s)
$$

\footnotetext{
${ }^{1}$ In the case of an open-channel, this can be achieved by a moveable hydraulic structure such as a weir or a gate, and $w$ represents the gate opening or the weir elevation.
}

with $G_{1}(s)=\frac{G(s)}{1-k_{h} \tilde{G}(s)}$ and $G_{2}(s)=\frac{k_{w} \tilde{G}(s)}{1-k_{h} \tilde{G}(s)}$. Then, for any $k_{h}>0$, the closed-loop system is stable (see [7]).

Now, the perfect rejection of the effect of measured upstream boundary perturbations on the downstream boundary is obtained by designing $w(s)=K_{F w}(s) q(s, 0)$ such that $h(s, L)=0$, i.e.:

$$
K_{F w}(s)=-\frac{1}{k_{w}} \frac{G(s)}{\tilde{G}(s)}=\frac{1}{k_{w}} K_{F}(s) .
$$

Therefore, for a perfect rejection, it is possible to compute $q(t, L)$ using the open-loop controller $K_{F}(s)$ and to implement it with $w(t)=\frac{q(t, L)}{k_{w}}$. This is equivalent to inverting eq. (8) by assuming that $h(s, L)=0$.

We focus below on the analysis of $K_{F}(s)$, keeping in mind that the implementation is done with a stabilizing feedback such as eq. (8).

\section{Series decomposition of the feedforward controller}

In the general case, it is necessary to use a rational approximation of $K_{F}(s)$ before implementation. One possibility to this end is to expand the transfer function into series, and then to truncate the infinite series.

The poles of the controller are the solutions of the following equation:

$$
e^{\left(\lambda_{2}(s)-\lambda_{1}(s)\right) L}=\frac{\lambda_{1}(s)}{\lambda_{2}(s)} .
$$

They can be shown to be stable, that is their real part is less than $-\epsilon$ with $\epsilon>0$, if and only if $\gamma \neq 0$ or $\delta \neq 0$.

Let us denote the poles $\left(p_{n}\right)_{n \in \mathbb{Z}}$, and $p_{-n}=\bar{p}_{n}$. Since each pole has single multiplicity, the rational approximation problem reduces to find the residues of $K_{F}(s)$ such that:

$$
K_{F}(s)=\sum_{n=-\infty}^{\infty} \frac{a_{n}}{s-p_{n}}
$$

with $a_{n}=\lim _{s \rightarrow p_{n}}\left(s-p_{n}\right) K_{F}(s)$, i.e.

$$
a_{n}=\left[\frac{\left(\lambda_{2}-\lambda_{1}\right) e^{\left(\lambda_{1}+\lambda_{2}\right) L}}{\left(1+L \lambda_{2}\right) \lambda_{2}^{\prime} e^{\lambda_{2} L}-\lambda_{1}^{\prime}\left(1+L \lambda_{1}\right) e^{\lambda_{1} L}}\right]\left(p_{n}\right)
$$

with

$$
\begin{aligned}
& \lambda_{i}^{\prime}(s)=\frac{(\alpha-\beta)}{2 \alpha \beta}+(-1)^{i} \frac{(\alpha+\beta)^{2} s+(\alpha-\beta) \gamma+2 \alpha \beta \delta}{2 \alpha \beta \sqrt{d(s)}} \\
& \text { and } d(s)=(\alpha+\beta)^{2} s^{2}+2[(\alpha-\beta) \gamma+2 \alpha \beta \delta] s+\gamma^{2} .
\end{aligned}
$$

D. Illustrative example in the case $\gamma=\delta=0$

In order to illustrate our approach and to link it with previous results presented by [1], we consider the special case where $\gamma=\delta=0$, corresponding to a frictionless horizontal canal. In this case, an analytical expression for open-loop control has been obtained by [1] using a Riemann invariants approach. Our formula (11) does not lead to the same expression. However, using the explicit frequency domain expression of the feedforward controller (7) and using another series decomposition, we recover the expression of [1]. Moreover, we derive upper and lower bounds for the $H_{\infty}$ norm of the approximation error due to the infinite series truncation. 
1) Explicit solution of the open-loop control: When $\gamma=$ $\delta=0$ the eigenvalues become $\lambda_{1}(s)=-\frac{s}{\alpha}$ and $\lambda_{2}(s)=\frac{s}{\beta}$.

Then, the open-loop controller $K_{F}(s)$ becomes:

$$
K_{F}(s)=\frac{(1+k) e^{-s \frac{L}{\alpha}}}{1+k e^{-\tau s}}
$$

where we have used the following notations:

$$
k=\frac{\beta}{\alpha}
$$

and

$$
\tau=\frac{L}{\alpha}+\frac{L}{\beta}
$$

This infinite dimensional controller can be approximated by a series of delays, using the well-known series expansion:

$$
\frac{1}{1+z}=\sum_{n=0}^{\infty}(-1)^{n} z^{n}
$$

which is valid for $|z|<1$.

In our case $0<k<1$ since $\beta<\alpha$, therefore the series converges and we get:

$$
K_{F}(s)=\sum_{n=0}^{\infty}(-1)^{n} k^{n}(1+k) e^{-s\left(\frac{L}{\alpha}+n \tau\right)}
$$

In the time domain, this expression leads to an explicit solution for the open-loop control:

$$
q(t, L)=\sum_{n=0}^{\infty}(-1)^{n} k^{n}(1+k) q\left(t-\frac{L}{\alpha}-n \tau, 0\right)
$$

This expression was first obtained by [1] using a Riemann invariants approach.

Our frequency domain approach allows to evaluate the approximation error generated by the truncation. We evaluate below the $H_{\infty}$ norm of the approximation error due to the truncation of the series (17).

2) Evaluation of the truncation error: When the infinite series (17) is truncated, this generates an approximation error which can easily be evaluated. Indeed, suppose that this series is approximated by the first $N$ elements of the series, and let us denote $K_{F, N}^{[1]}(s)$ this truncated series. We have:

$$
K_{F, N}^{[1]}(s)=\sum_{n=0}^{N}(-1)^{n} k^{n}(1+k) e^{-j \omega\left(\frac{L}{\alpha}+n \tau\right)}
$$

Then, let us evaluate the norm of the approximation error for $s=j \omega$

$$
\begin{aligned}
\left|K_{F}(j \omega)-K_{F, N}^{[1]}(j \omega)\right| & =(1+k)\left|\sum_{n=N+1}^{\infty}(-1)^{n} k^{n} e^{-j \omega\left(\frac{L}{\alpha}+n \tau\right)}\right| \\
& =\left|\frac{(1+k) k^{N+1} e^{-j \omega\left(\frac{L}{\alpha}+(N+1) \tau\right)}}{1+k e^{-\tau j \omega}}\right| \\
& =\frac{(1+k) k^{N+1}}{\sqrt{1+k^{2}+2 k \cos (\omega \tau)}}
\end{aligned}
$$

Since $|\cos (\omega \tau)| \leq 1$, we have the double inequality:

$$
k^{N+1} \leq\left|K_{F}(j \omega)-K_{F, N}^{[1]}(j \omega)\right| \leq \frac{(1+k)}{1-k} k^{N+1}
$$

Therefore the worst case approximation error $\left(H_{\infty}\right.$ norm) will always be larger than $k^{N+1}$. Since $k<1$, this tends towards zero as $N$ tends towards $\infty$. This convergence can however be slow especially for canals where $\alpha$ and $\beta$ are very close.

Remark 1 (Delay-free controller): There are various possibilities for approximating the feedforward controller (e.g. Eqs. (11) or (17)), but the series may not converge very rapidly. It may be necessary to use a large number of terms in order to get a good approximation. As we will show in the application, a better approximation can be achieved by extracting the delay $\exp \left(-\frac{L}{\alpha} s\right)$ from the controller and considering the delay-free controller:

$$
\tilde{K}_{F}(s)=K_{F}(s) e^{\frac{L}{\alpha} s}
$$

In this case, a good low frequency approximate solution of $\operatorname{order} N$ is obtained by:

$$
\tilde{K}_{F, N}(s)=1+\sum_{n=-N}^{N}\left(\frac{\tilde{a}_{n}}{s-p_{n}}+\frac{\tilde{a}_{n}}{p_{n}}\right)
$$

with $\tilde{a}_{n}=\lim _{s \rightarrow p_{n}}\left(s-p_{n}\right) \tilde{K}_{F}(s)$, i.e.

$$
\tilde{a}_{n}=\left[\frac{\left(\lambda_{2}-\lambda_{1}\right) e^{\left(\lambda_{1}+\lambda_{2}+\frac{s}{\alpha}\right) L}}{\left(1+L \lambda_{2}\right) \lambda_{2}^{\prime} e^{\lambda_{2} L}-\lambda_{1}^{\prime}\left(1+L \lambda_{1}\right) e^{\lambda_{1} L}}\right]\left(p_{n}\right)
$$

\section{E. Rational approximation}

The above approaches have considered series decomposition of the feedforward controller, with a given order, leading to a given truncation error. Another interesting possibility is to consider a finite bandwidth approximation of the controller, with a bounded error for higher frequencies. This is coherent with the fact that the control is implemented by finite bandwidth actuators. In this case, the problem can be stated as an $H_{\infty}$-like minimization one, where one tries to minimize the norm of the difference between the feedforward controller $K_{F}(s)$ and its rational approximation $K_{F A}(s)$ :

$$
\min \left|K_{F}(j \omega)-K_{F A}(j \omega)\right| \text { for } \omega \in\left[0, \omega_{0}\right]
$$

and

$$
\left|K_{F}(j \omega)-K_{F A}(j \omega)\right|<K \text { for } \omega>\omega_{0}
$$

This problem is a convex one that has already been solved [8]. But this approach is rather naive, since the frequency bandwidth of interest remains to be determined, and the system is uncertain in high frequencies. In order to take into account these uncertainties, it is more realistic to directly consider the robust feedforward control problem, as we do in the following section.

\section{ROBUST FEEDFORWARD DESIGN}

The previous approach has two possible drawbacks: $(i)$ the plant model is assumed to be perfect, $(i i)$ the irrational controller has to be approximated by a rational one. A remedy to these drawback is to define an uncertain model, express the feedforward control problem as an $H_{\infty}$ criterium and then apply the robust $H_{\infty}$ feedforward control approach proposed in [11]. 
Let us denote by $\star$ the Redheffer product. The first point is that the plant can be modelled as $\Delta \star G$ where $\Delta$ is the uncertainty which is stable such that $\|\Delta\|_{\infty}<1$ and where $G$ is a rational transfer function matrix, with two inputs and two outputs. The introduction of $\Delta$ allows to rigourously take into account $(i)$ the approximation of the irrational plant by a rational transfer function, (ii) the model uncertainty.

In the sequel, the control input, the controlled output and the perturbation are denoted $u, y$ and $d$. Let us introduce $W_{y}$ and $W_{u}$ two weighting transfer functions. $W_{y}$ allows to define the considered disturbance and the rejection performance (rejection time, asymptotic rejection). $W_{u}$ allows to limit the control input $u$. The feedforward problem can be written as the following weighted $H_{\infty}$ norm minimization (see Figure 1) : Find the stable rational transfer function $K_{f}$ such that

$$
\sup _{\Delta,\|\Delta\|_{\infty}<1}\left\|\left[\begin{array}{c}
W_{y} \times(\Delta \star G) \\
W_{u}
\end{array}\right] K_{f}\right\|_{\infty}<1 .
$$

This problem is a subcase of the general robust $H_{\infty}$

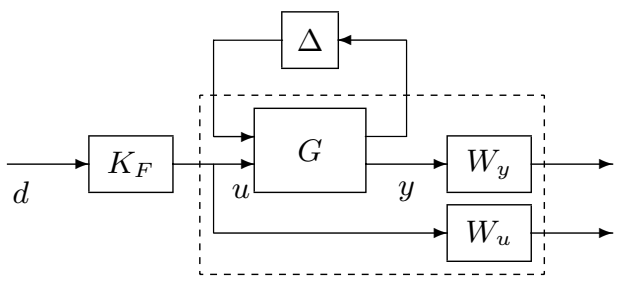

Fig. 1. Robust $H_{\infty}$ feedforward control problem

feedforward control problem (see Figure 2) considered in [11]. In the sequel, we present an application of Theorem

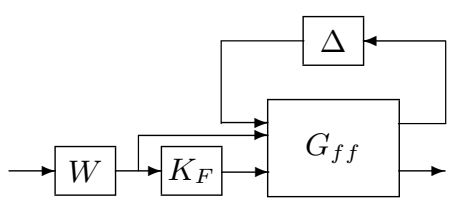

Fig. 2. General robust $H_{\infty}$ feedforward control problem

\section{1 presented in [11].}

With $N>0$ a given integer, let $\mathbf{X}(\boldsymbol{\Delta})$ be the set of $N \times N$ real symmetric matrices of the structure:

$$
\left[\begin{array}{cccccc}
2 x_{0} & 0 & x_{1} & 0 & \cdots & \cdots \\
0 & 0 & \cdots & \cdots & 0 & \vdots \\
\vdots & \vdots & \ddots & \ddots & \vdots & x_{N-1} \\
\vdots & 0 & \ddots & \ddots & \vdots & 0 \\
\vdots & \cdots & \cdots & x_{N-1} & 0 & 2 x_{N}
\end{array}\right] .
$$

Let $B(s)$ be $\left[\begin{array}{llll}s^{N} & s^{N-1} & \cdots & 1\end{array}\right]^{T}$ and $d(s)=s^{N}+$ $d_{1} s^{N-1}+\cdots+d_{0}$ a given polynomial with roots whose real parts are strictly negative. The state space representation of $\frac{B(s)}{d(s)}$ and

$$
\left[\begin{array}{cc}
{\left[\begin{array}{cc}
I & 0 \\
0 & W_{y}(s)
\end{array}\right] G(s)} \\
0 & W_{u}(s)
\end{array}\right]
$$

are denoted:

$$
\left[\begin{array}{c|c}
A_{b} & B_{b} \\
\hline C_{b} & D_{b}
\end{array}\right] \quad \text { and } \quad\left[\begin{array}{c|cc}
A^{p} & B_{1} & B_{2} \\
\hline C^{p} & D_{1} & D_{2}
\end{array}\right] .
$$

Theorem 1: The robust $H_{\infty}$ feedforward control problem has a solution if and only if there exist $N>0$, matrices $P$, $Z, X \in \mathbf{X}(\boldsymbol{\Delta}), \mathbf{A}, \mathbf{B}, \mathbf{C}$ and $\mathbf{D}$ such that

(i) $P>Z, Z>0$ and

$$
\left[\begin{array}{c|c}
\mathcal{L}_{1}(P, Z, \mathbf{A}, \mathbf{C})+\cdots & \mathcal{L}_{3}(\mathbf{B}, \mathbf{D})^{T} \\
\mathcal{L}_{1}(P, Z, \mathbf{A}, \mathbf{C})^{T}+\mathcal{L}_{2}(X) & -I
\end{array}\right]<0
$$

with $\mathcal{L}_{1}(P, Z, \mathbf{A}, \mathbf{C})$ defined by:

$$
\begin{aligned}
& {\left[\begin{array}{c}
P \\
P-Z \\
0
\end{array}\right]\left[\begin{array}{ccc|c|l}
A_{b} & 0 & 0 & 0 & {\left[\begin{array}{ll}
B_{b} & 0
\end{array}\right]} \\
0 & A_{b} & B_{b} B_{1}^{T} & 0 & B_{b} D_{1}^{T} \\
0 & 0 & A^{T} & 0 & C^{T}
\end{array}\right]} \\
& \cdots+\left[\begin{array}{c}
I \\
I \\
0
\end{array}\right]\left[\begin{array}{ll}
\mathbf{A}^{T} & \mathbf{C}^{T}
\end{array}\right]\left[\begin{array}{ccc|c|c}
0 & 0 & 0 & I & 0 \\
0 & 0 & B_{2}{ }^{T} & 0 & D_{2}^{T}
\end{array}\right] \\
& \mathcal{L}_{2}(X)=\Omega^{T}\left[\begin{array}{ccc}
X & 0 & 0 \\
0 & -X & 0 \\
0 & 0 & -\gamma^{2} I
\end{array}\right] \Omega
\end{aligned}
$$

with $\Omega$ defined by:

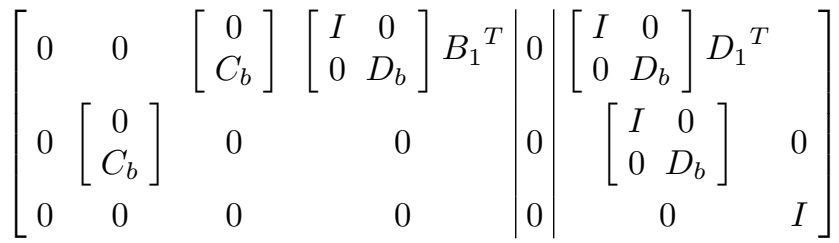

and $\mathcal{L}_{3}(\mathbf{B}, \mathbf{D})$ is defined by

$$
\left[\begin{array}{cc}
\mathbf{B}^{T} & \mathbf{D}^{T}
\end{array}\right]\left[\begin{array}{cccc|c|c}
0 & 0 & 0 & 0 & I & 0 \\
0 & 0 & 0 & B_{2}{ }^{T} & 0 & D_{2}^{T}
\end{array}\right] .
$$

(ii) $P_{b}=P_{b}^{T}$ and

$$
\left[\begin{array}{cc}
A_{b}^{T} P_{b}+P_{b} A_{b} & P_{b} B_{b} \\
B_{b}^{T} P_{b}^{T} & 0
\end{array}\right]+\left[\begin{array}{c}
C_{b}^{T} \\
D_{b}^{T}
\end{array}\right] X\left[\begin{array}{ll}
C_{b} & D_{b}
\end{array}\right]>0
$$

Testing Theorem 1 condition is a feasibility problem involving Linear Matrix Inequality constraints which can be efficiently solved [3]. If the condition is satisfied then the robust feedforward $K_{F}$ is obtained as

$$
K_{F}(s)=\left(\mathbf{D}+\mathbf{C}(s(P-Z)-\mathbf{A})^{-1} \mathbf{B}\right) .
$$


The proof of the sufficiency is obtained by application of Theorem 5.1 presented in [11]. Necessity is obtained as the $\mu$ upper bound is equal to the actual value of $\mu$ in the case of one dynamical uncertainty [10], with the choice of a multiplier/scaling whose degree $N$ is large enough [4].

\section{APPLICATION TO AN OPEN-CHANNEL}

\section{A. Saint-Venant equations}

We consider a prismatic canal pool of length $L$ with uniform geometry (not necessarily rectangular) and a given slope $S_{b} \geq 0$. The Saint-Venant equations are hyperbolic nonlinear partial differential equations involving the average discharge $Q(t, x)$ and the water depth $H(t, x)$ along one space dimension [5]:

$$
\begin{aligned}
\frac{\partial A}{\partial t}+\frac{\partial Q}{\partial x} & =0 \\
\frac{\partial Q}{\partial t}+\frac{\partial Q^{2} / A}{\partial x}+g A \frac{\partial H}{\partial x} & =g A\left(S_{b}-\frac{Q^{2} n^{2}}{A^{2} R^{4 / 3}}\right)
\end{aligned}
$$

where $A(t, x)$ is the wetted area $\left(\mathrm{m}^{2}\right), Q(t, x)$ the discharge $\left(\mathrm{m}^{3} / \mathrm{s}\right)$ across section $A, V(t, x)$ the average velocity $(\mathrm{m} / \mathrm{s})$ in section $A, H(t, x)$ the water depth (m), $g$ the gravitational acceleration $\left(\mathrm{m} / \mathrm{s}^{2}\right), n$ the Manning coefficient $\left(\mathrm{sm}^{-1 / 3}\right)$ and $R$ the hydraulic radius (m), defined by $R=A / P$, where $P$ is the wetted perimeter $(\mathrm{m})$.

The boundary conditions are $Q(0, t)=Q_{0}(t)$ and $Q(L, t)=Q_{L}(t)$. These boundary conditions are suited for control purposes, since the system is then combined with gates linking locally the discharge with the water elevation. The initial conditions are given by $Q(0, x)$ and $H(0, x)$.

\section{B. Linearized Saint-Venant equations}

We consider small variations of discharge $q(t, x)$ and water depth $h(t, x)$ around constant stationary values $Q_{0}\left(\mathrm{~m}^{3} / \mathrm{s}\right)$ and $H_{0}(\mathrm{~m})$. When $S_{b} \neq 0$, the equilibrium regime $\left(H_{0}, Q_{0}\right)$ verifies the following algebraic equation:

$$
S_{b}=\frac{Q_{0}^{2} n^{2}}{A_{0}^{2} R_{0}^{4 / 3}}
$$

If the slope $S_{b}$ is zero and $n=0$, then any couple $\left(H_{0}, Q_{0}\right)$ can be chosen as an equilibrium solution, provided that the Froude number $F_{0}=V_{0} / C_{0}$ remains strictly lower than $1 . V_{0}$ is the average velocity $(\mathrm{m} / \mathrm{s})$ and $C_{0}=\sqrt{g A_{0} / T_{0}}$ the wave celerity $(\mathrm{m} / \mathrm{s})$, with $T_{0}$ the water surface top width $(\mathrm{m})$.

Linearizing the Saint-Venant equations around these stationary values leads to a linear hyperbolic system of partial differential equations (1) with the following values of the constant parameters:

$$
\begin{aligned}
\alpha & =C_{0}+V_{0} \\
\beta & =C_{0}-V_{0} \\
\gamma & =g S_{b}\left(\frac{10}{3}-\frac{4 A_{0}}{3 T_{0} P_{0}} \frac{d P_{0}}{d H}\right) \\
\delta & =\frac{2 g S_{b}}{V_{0}}
\end{aligned}
$$

Note that the variable $h$ is scaled by a factor $T_{0}$, i.e. eq. (1) applies in fact to $h^{*}=T_{0} h$, which is denoted $h$ with an abuse of notation.

\section{Closed-loop controller}

The stabilizing proportional boundary control is performed with a moveable weir, defined by the linearized equation:

$$
q(s, L)=k_{h} h(s, L)+k_{w} w(s)
$$

with $k_{h}=0.6 \sqrt{2 g} L_{w}$, where $L_{w}$ is the length of the weir, and $k_{w}=-k_{h}$. In the simulations, the weir length is equal to $0.5 T_{0}$.

\section{Feedforward controller}

1) Bode diagrams: The feedforward controller is obtained following Eq. (7). Figure 3 depicts the Bode diagram of controller $K_{F}(s)$ for an hyperbolic system with the following parameters $\alpha=4.63, \beta=3.33, \gamma=2.7 \times 10^{-3}, \delta=$ $3 \times 10^{-3}$ and $L=3000 \mathrm{~m}$. These parameters correspond to the linearized Saint-Venant representing a canal pool with a trapezoidal geometry, (bed width of $7 \mathrm{~m}$, side slope of 1.5), a bed slope $S_{b}=0.0001$ and Manning coefficient of 0.02 . The considered stationary regime corresponds to a discharge $Q_{0}=14 \mathrm{~m}^{3} / \mathrm{s}$ and a water depth $H_{0}=2.12 \mathrm{~m}$.
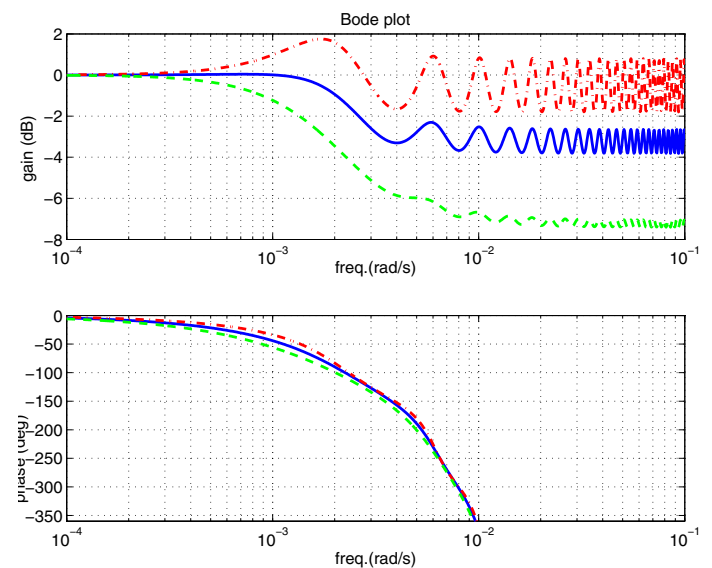

Fig. 3. Bode plot of $K_{F}(s)$ with $(\alpha, \beta, \gamma, \delta)=(4.63,3.33,2.7 \times$ $\left.10^{-3}, 3 \times 10^{-3}\right)$ (solid blue). The two others plots are obtained with parameters $\gamma$ and $\delta$ changed by $\pm 50 \%: \gamma_{1}=\gamma / 1.5, \delta_{1}=\delta / 1.5$ (dashdotted red), and $\gamma_{2}=1.5 \gamma, \delta_{2}=1.5 \delta$ (dashed green).

Figure 3 compares the feedforward controllers $K_{F}(s)$ for three canals with the same length, same $\alpha$ and $\beta$, but with different slope and friction, leading to different $\gamma$ and $\delta$. It is clear from Figure 3 that even small changes of the slope and the friction dramatically change the Bode diagram of the feedforward controller. In practise, such a sensitivity is not acceptable. It is therefore necessary to take account of these elements in the feedforward control design.

\section{2) Rational approximations:}

a) Complete controller: The rational approximations obtained with eq. (11) are compared to $K_{F}(s)$ on figure 4. The Bode diagram shows that a higher number of poles gives a better approximation of the controller. 

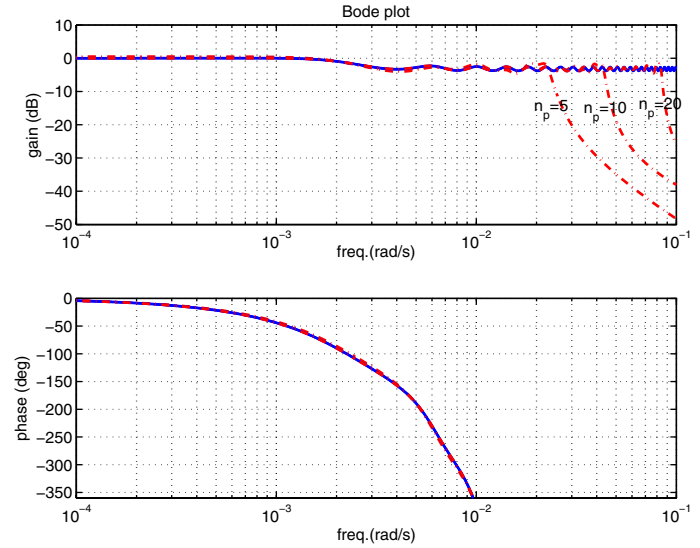

Fig. 4. Bode plot of $K_{F}(s)$ (solid blue) and rational approximations obtained with eq. (11), using 5, 10 and 20 poles (dash-dotted red).

b) Reduced controller: We compared the rational approximations obtained with eq. (19) to $K_{F}(s)$ (results not displayed for lack of space). The Bode plot shows that a higher number of poles gives a better approximation of the controller. In this case, a good approximation is obtained with only 2 pair of poles, leading to a delay-free controller of dimension 4.

We also observe that with the same number of poles, the delay-free controller (19) leads to a better approximation of $K_{F}(s)$ than the one including the delay (11).

3) Simulation results: Figure 5 compares the water level $h(t, L)$ and the feedforward discharge $q(t, L)$ obtained with controller $\tilde{K}_{F}(s)$ with 5 poles and the controller of [1] obtained with Eq. (18). It is clear from Figure 5 that a controller designed by assuming $\delta=\gamma=0$ gives large poorly damped oscillations of the control $q(t, L)$, leading to a large error in the downstream water level. On the contrary, when the damping linked to the nonzero slope and friction is taken into account, the control $q(t, L)$ is much more smooth and the output error much smaller.
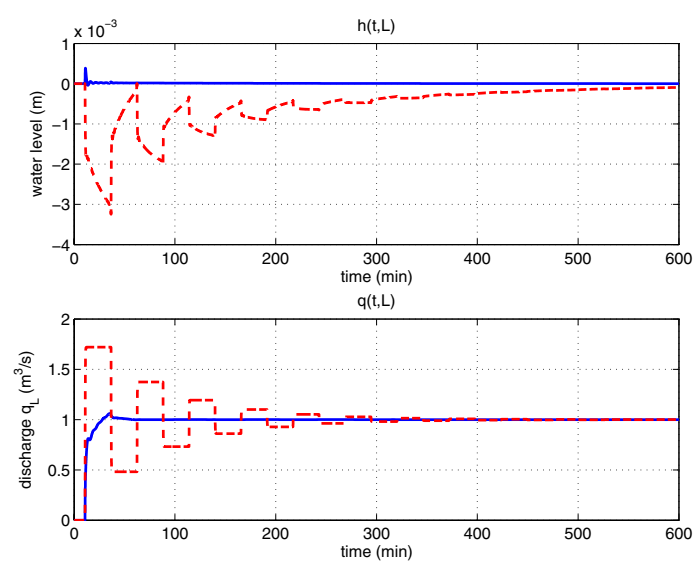

Fig. 5. Downstream water level error $h(t, L)$ and feedforward control $q(t, L)$ for a rational approximations of $\tilde{K}_{F}$ using 5 poles (solid blue) and the controller of [1] (dashed red).
This is also clear from the Bode plot of figure 6 .
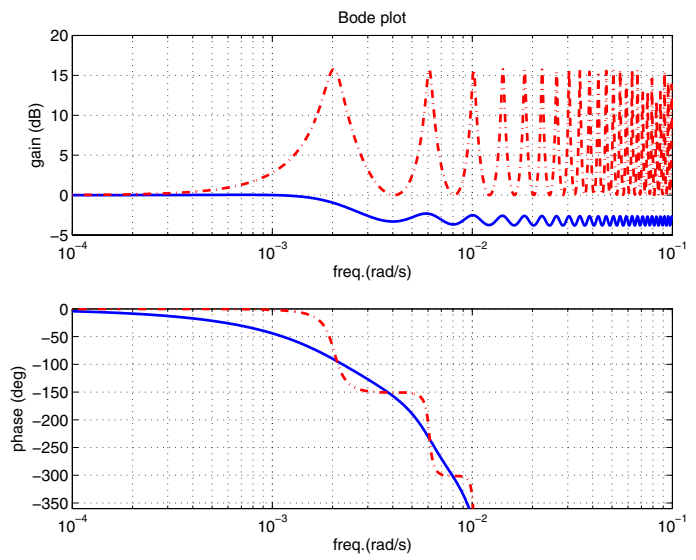

Fig. 6. Bode diagram of $K_{F}(s)$ (solid blue) and of the controller of [1] (dash-dotted red).

\section{CONCLUSION}

The paper has used a frequency domain approach to design a feedforward boundary controller to reject measured perturbations on a plant whose model is a linear hyperbolic equation derived from two conservation laws. The result of [1] is obtained as a special case of our result. A robust feedforward controller design is also proposed, to take account of the model uncertainties in high frequencies. Frequency domain and time domain comparisons have shown the performance of the feedforward controller design.

\section{REFERENCES}

[1] G. Bastin, J.M. Coron, B. D’Andréa-Novel, and L. Moens. Boundary control for exact cancellation of boundary disturbances in hyperbolic systems of conservation laws. In $44^{\text {th }}$ Conf. on Decision and Control, pp. 1086-1089, Sevilla, 2005.

[2] E. Bautista and A. J. Clemmens. Response of ASCE task committee test cases to open-loop control measures. J. Irrig. Drain. Engrg., 125(4):179-188, July/August 1999.

[3] S. Boyd, L. El Ghaoui, E. Feron, and V. Balakrishnan. Linear Matrix Inequalities in Systems and Control Theory, volume 15 of Studies in Appl. Math. SIAM, Philadelphia, June 1994.

[4] Y.-S. Chou, A.L. Tits, and V. Balakrishnan. Stability multipliers and $\mu$ upper bounds: connections and implications for numerical verification of frequency domain conditions. IEEE Trans. Aut. Control, 44(5):906913, 1999.

[5] V.T. Chow. Open-channel Hydraulics. McGraw-Hill Book Company, New York, 1988. 680 p.

[6] X. Litrico and V. Fromion. Frequency modeling of open channel flow. J. Hydraul. Engrg., 130(8):806-815, 2004.

[7] X. Litrico and V. Fromion. Boundary control of linearized SaintVenant equations oscillating modes. Automatica, 42(6):967-972, 2006.

[8] X. Litrico and V. Fromion. $H_{\infty}$ control of an irrigation canal pool with a mixed control politics. IEEE Trans. on Control Systems Technology, 14(1):99-111, 2006.

[9] Fubo Liu, Jan Feyen, P.-O. Malaterre, J.-P. Baume, and P. Kosuth. Development and evaluation of canal automation algorithm CLIS. $J$. Irrig. Drain. Engrg., 124(1):40-46, 1998.

[10] A. Packard and J.C. Doyle. The complex structured singular value. Automatica, 29(1):71-109, 1993.

[11] G. Scorletti and V. Fromion. Further results on the design of robust $H_{\infty}$ feedforward controllers and filters. Conf. on Decision and Control, 2006. 